\title{
A Percepção da Comunidade Sobre a Atuação do Serviço de Atenção Farmacêutica em Ações de Educação em Saúde Relacionadas à Promoção do Uso Racional de Medicamentos' Community's Perception Towards the Performance of Pharmaceutical Service Care in the Health Education Actions Related to Rational Medicine Use
}

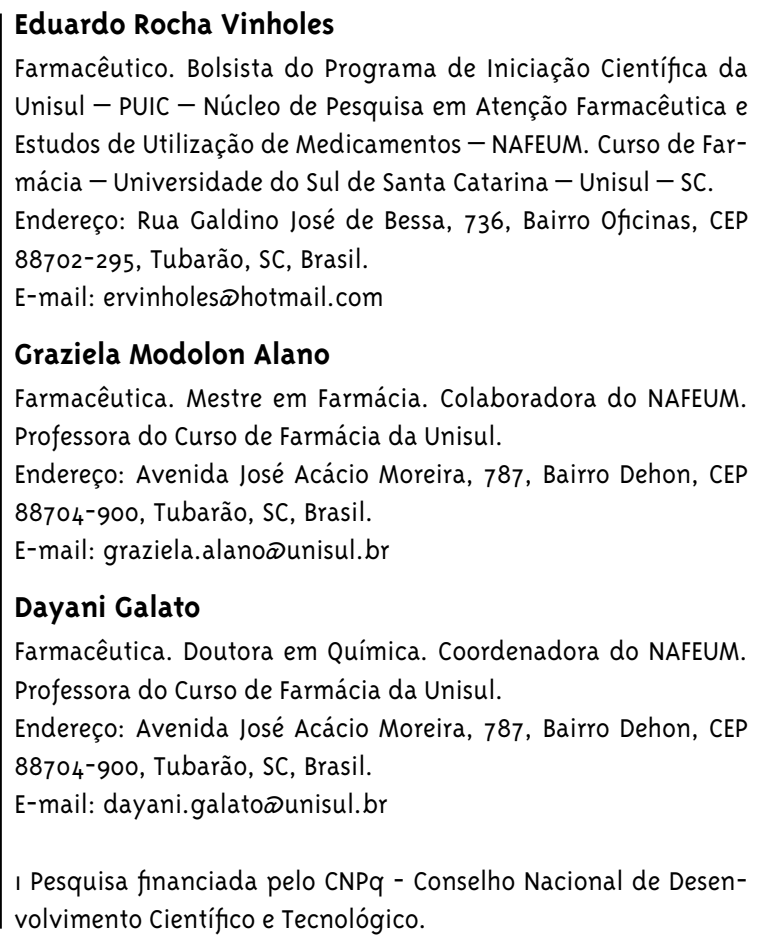

\section{Resumo}

A educação em saúde visa democratizar o acesso ao conhecimento e tornar os indivíduos da comunidade capazes de atuar como corresponsáveis na promoção da saúde. 0 objetivo deste trabalho foi apresentar a experiência do Serviço de Atenção Farmacêutica em ações na comunidade com vistas a promover o uso racional de medicamentos. Utilizou-se uma abordagem qualitativa, do tipo pesquisa-ação. Realizou-se a descrição da experiência e o levantamento das percepções dos responsáveis pelos grupos envolvidos nas palestras. Foram realizadas 22 palestras alcançando 565 participantes; os temas pré-selecionados envolviam assuntos como polimedicação, automedicação e adesão terapêutica, e os que surgiram no decorrer das palestras foram classificados como temas emergentes, representados por experiências dos participantes quanto às doenças e suas formas de tratamento, os efeitos colaterais de medicamentos, o manejo não farmacológico de problemas de saúde, qualidade, segurança e eficácia de medicamentos genéricos e o acesso aos medicamentos. Na percepção dos líderes dos grupos foi mencionado que as palestras contribuíram para o uso racional de medicamentos, proporcionando a mudança de postura, segundo alguns relatos. As palestras colaboraram para reforçar que o papel da equipe de saúde não é apenas o de permitir o acesso aos medicamentos, mas também de garantir o seu uso correto. Essa experiência destaca a ação do farmacêutico como profissional da saúde e o papel que desempenha na promoção do uso racional de medicamentos, empregando nesta ação, além do 
conhecimento científico, habilidades para usar as experiências da população no ato de ensinar, respeitando o indivíduo e tornando-o ativo nesse processo.

Palavras-chave: Educação em saúde; Uso de medicamentos; Assistência farmacêutica.

\section{Abstract}

Health education aims at democratizing the access to knowledge and to make the community people able to act as co-managers in health's promotion. This work's objective was to present pharmaceutical service care experience in action in the community, in order to promote the rational use of medicine. We used a research- action qualitative approach. Experience description and people's perception survey (responsible for groups involved in the current lecture) were accomplished, and observed in this study. 22 lectures were done, reaching as a total 565 participants; preselected themes involved subjects as polymedication, self-medication and adhesion therapeutics, and the ones that emerged during each lecture were classified as emergent topics, represented by participant experiences (as for their diseases and treatment solutions, their collateral effects etc; also non-pharmacological health problems handling; quality, security and generic medicine effectiveness and medicine access should be approached here, in these cases). under group leaders perception, and approval,; it was mentioned that the lectures contributed to a more rational use, provided an important posture change, as some reports in the media said, as can be read. The lectures collaborated to reinforce that the health team role is not only to allow an access to medicine, but also to guarantee its correct use. This experience highlights the pharmacist action as a health professional. His role is carried out in rational medicine use promotion, employing in this action -- besides scientific knowledge -- abilities to use the population experiences in the action of teaching, respecting all individuals and making them active in this process.

Keywords: Health Education; Drug Utilization; Pharmaceutical Care.

\section{Introdução}

O processo de educação em saúde tem como objetivo principal preparar os membros de uma comunidade para atuarem como corresponsáveis na promoção da saúde, através da interface entre saberes científicos, senso comum e as experiências vivenciadas, de maneira a promover continuamente a integração, a continuidade, a democratização do conhecimento e o avanço na área social (Lima e col., 200o).

Nesse contexto, a promoção da saúde pode ser entendida como uma ferramenta de preparação da comunidade para contribuir na melhoria da sua qualidade de vida e saúde, exigindo o comprometimento de seus integrantes na condução desse processo (Machado e col., 2007).

Vários são os conceitos e fundamentos que sustentam a prática da educação e da promoção em saúde. A educação em saúde busca provocar modificações de comportamento individual, enquanto que a promoção em saúde pretende gerar modificações de comportamento organizacional, suficientes para beneficiar e abranger a saúde de camadas mais amplas da população (Candeias, 1997). A educação em saúde consiste em um componente da promoção de saúde, porém é muito mais específica, tendo como meta formar indivíduos mais cientes dos fatores que podem ajudá-los a manter uma boa saúde e prevenir doenças. A informação sobre o uso adequado de medicamentos é uma face importante da educação em saúde, porém há muitas outras situações em que esse serviço pode ser prestado (Winfield e Richards, 1998).

A educação em saúde envolve um processo que extrapola os limites das informações relativas a uma determinada prescrição. Educar implica em algo mais do que a simples divulgação de conhecimento, deve envolver processos que contribuam para a mudança de atitude e mesmo de conduta das pessoas. Por meio desse processo, é possível, portanto, demover indivíduos de pressupostos equivocados e concepções errôneas (Marin e col., 2003).

Destaca-se que, nesse processo, a população tem a opção de aceitar ou rejeitar as novas informações, podendo, também, adotar ou não novos comportamentos frente aos problemas de saúde. Portanto, não basta apenas seguir as recomendações para melhorar a saúde e prevenir doenças, mas também efetivar a educação em saúde num processo que estimule o diálogo, a inda- 
gação, a reflexão, o questionamento e a ação partilhada (Martins e col., 2007).

Ressalta-se que a educação em saúde é um método para promovê-la neste processo de capacitação individual e coletiva de responsabilidade e de direitos, incentivando ações que sigam os princípios do sistema público. A partir desse ponto de vista, escolher formas didáticas que levem a uma modificação dos indivíduos integrantes da sociedade, expandindo sua habilidade de percepção dos diversos fatores determinantes do conceito de ser saudável é fundamental para a obtenção de resultados (Machado e col., 2007).

Portanto, a conscientização da comunidade é um pré-requisito quando se espera alcançar elevado nível de saúde. Entre os diversos fatores que influenciam no estabelecimento e manutenção da saúde do indivíduo, o medicamento está envolvido em grande parte dessas atividades. Dessa forma, torna-se necessário que a população esteja orientada sobre como proceder em relação ao uso de medicamentos, para que estes tenham uma ação efetiva e segura. A abrangência do processo de educação permite abordar inúmeros aspectos, como a conservação, a automedicação, grupos de risco, gravidez e lactação, bebidas alcoólicas, prazo de validade e outros (Marin e col., 2003).

\section{0 uso racional de medicamentos}

A Organização Mundial de Saúde define o uso racional de medicamentos como sendo a situação em que "o paciente recebe o medicamento apropriado a sua necessidade clínica, na dose e posologia corretas, por um período de tempo adequado e ao menor custo para si e para a comunidade" (OMS, 2002, p. 1).

$\mathrm{O}$ uso racional de medicamentos inclui alguns aspectos que corroboram com o preconizado pela Política Nacional de Medicamentos, conforme segue: seleção adequada da terapêutica, incluindo a indicação (dose, posologia e duração do tratamento) e o medicamento apropriado à situação clínica do paciente; garantia de eficácia, segurança e conveniência para o mesmo. Acrescenta-se, ainda, a importância de dispensação correta dos medicamentos a serem utilizados pelo paciente e os repasses das informações necessárias para garantir a adesão ao tratamento pelo paciente, o prosseguimento dos efeitos desejados e a identificação de possíveis eventos adversos decorrentes do tratamento (Marin e col., 2003).
O crescimento da indústria farmacêutica fomentou a criação de novas necessidades de consumo de medicamentos como compensação indispensável ao seu desenvolvimento. Aconteceu, em razão disso, uma alteração dos hábitos de consumo e das práticas dos profissionais e das instituições de saúde. A propaganda de medicamentos instituiu conceitos relacionados à sua eficácia, com sustentação científica e a simultânea dificuldade em evitar seu uso, que começou a orientar práticas que procuravam a preservação ou a restituição da saúde (Naves e col., 2005, p. 1006), surgindo, dessa forma, uma sociedade medicalizada (Barros, 2004).

Atualmente, no Brasil, coexistem, por um lado, a dificuldade de acesso de parcela significativa da população aos medicamentos essenciais e, por outro, o uso abusivo e irracional pelas classes que têm maior poder de compra (Naves e col., 2005).

Segundo a Organização Mundial de Saúde (2002), entre as intervenções fundamentais para a promoção do uso racional de medicamentos está a educação da população a respeito dos medicamentos, a qual pode estar associada à Atenção Farmacêutica.

O processo de promoção da Atenção Farmacêutica no país foi apoiado pela Organização Pan--Americana de Saúde (OPAS) e pela Organização Mundial de Saúde (OMS) e envolveu pesquisadores, formuladores de políticas, entidades e diversos profissionais. Ainda que sua definição continue em uma discussão terminológica intensa em cada país, é interessante ressaltar que, no Brasil, o termo Atenção Farmacêutica considera a promoção da saúde, incluindo a educação em saúde, como componente do conceito, o que constitui um diferencial marcante em relação ao conceito adotado em outros países (OPAS, 2002), sendo este incentivado pela Organização Mundial de Saúde (OMS, 1993).

A Organização Mundial de Saúde (OMS, 1993) conceitua a Atenção Farmacêutica como uma prática profissional em que o paciente é o principal beneficiário das ações do farmacêutico. Essa prática profissional compreende um conjunto de atitudes, comportamentos, compromissos, cuidados, valores éticos, funções, conhecimentos, responsabilidades e habilidades do farmacêutico na prestação da farmacoterapia, com o objetivo de obter resultados terapêuticos sustentados pelos indicadores de saúde e de qualidade de vida dos pacientes. Nesse contexto, mesmo que centralizada 
na farmacoterapia, a OMS reconhece o farmacêutico como educador que pode contribuir na prevenção de doenças e na promoção da saúde com outros membros numa equipe multiprofissional.

O Serviço de Atenção Farmacêutica (SAF) foi criado pelo Curso de Farmácia da Universidade do Sul de Santa Catarina, em 2006, e atualmente funciona em uma clínica escola multidisciplinar. Suas ações são voltadas para o uso racional de medicamentos, sendo desenvolvidas com a comunidade. Essas ações podem ser abrangentes como palestras a grupos de pacientes previamente constituídos, ou envolver atendimentos individuais, que têm como foco principal as orientações sobre o uso correto de medicamentos utilizados pelos pacientes. Este artigo tem como objetivo apresentar a experiência do SAF em ações de educação em saúde desenvolvidas junto a grupos de indivíduos, visando à promoção do uso racional de medicamentos e à compreensão da opinião dos participantes em relação a essas atividades.

\section{Percurso Metodológico: a experiência do SAF}

Este relato segue uma abordagem qualitativa, do tipo pesquisa-ação (Minayo, 2004), na descrição da experiência do SAF. A entrada em campo foi realizada inicialmente por meio de contato com os coordenadores de grupo e levantamentos das expectativas. 0 trabalho em campo foi consolidado com a realização das palestras, com os registros realizados pelos proponentes ao final de cada evento (notas do pesquisador) e com entrevistas com os responsáveis pelos grupos. Por último, foram analisados os dados com a sistematização das notas do pesquisador e das percepções dos envolvidos no processo.

A experiência descrita neste trabalho está dividida em dois momentos, sendo o primeiro representado por ações, e o segundo pelas percepções de pessoas responsáveis pelos grupos contemplados pelas ações do SAF.

\section{As ações}

Todas as ações de educação em saúde foram realizadas com grupos estabelecidos em comunidades da Associação dos Municípios da Região de Laguna (AMUREL), onde a Universidade está localizada.
As ações de educação em saúde iniciaram-se através de um primeiro contato mantido com os coordenadores ou responsáveis de grupos preeexistentes, entre os quais o de pacientes com hipertensão e diabetes, grupos da terceira idade e grupos de mulheres, além de pessoas vinculadas à Secretaria de Assistência Social da prefeitura e a postos de saúde da rede pública, para verificar o interesse e a necessidade da realização de palestras sobre temas referentes ao uso racional de medicamentos.

A partir desse primeiro contato foram definidos os temas que seriam apresentados aos grupos, em conjunto com cada responsável pelos mesmos, de acordo com as particularidades e necessidades consideradas mais relevantes. Os temas pré-selecionados foram referentes ao uso racional de medicamentos, como polimedicação, automedicação na terceira idade, manejo de doenças crônicas (hipertensão, diabetes e dislipidemias), adesão terapêutica e cuidados com o uso e a conservação de medicamentos.

Os diapositivos utilizados para conduzir as apresentações foram elaborados pelos profissionais farmacêuticos professores da universidade, contando com o auxílio de acadêmicos do curso de Farmácia, utilizando como fundamentação teórica dados retirados da literatura, de artigos científicos e de pesquisas sobre utilização de medicamentos realizadas na comunidade da AMUREL pela própria Universidade.

As palestras foram apresentadas por farmacêuticos professores do curso de Farmácia da Universidade, com a participação, em alguns momentos, de acadêmicos do mesmo curso. Não houve treinamento formal dos expositores, no entanto, durante a elaboração dos diapositivos e da discussão dos tópicos era combinado entre os palestrantes como seria a abordagem dos temas definidos.

Nas intervenções em grupo seguiu-se um modelo interativo, sendo adotada uma apresentação expositiva e dialogada, onde os participantes foram estimulados a relatar as suas experiências vivenciadas com os tratamentos e a apresentar as suas dúvidas quanto ao uso dos medicamentos. Foi observado que essa mesma estratégia de atuação foi apresentada por outros pesquisadores (Silva e col., 2006). Portanto, como as atividades de educação em saúde foram realizadas de forma expositiva, possibilitaram aos sujeitos uma participação ativa, através do diálogo direto com o profis- 
sional farmacêutico, formulação de questionamentos e intervenções a qualquer momento do evento.

Em algumas situações, mesmo com a disponibilidade do equipamento para projeção visual, optou-se apenas pela explanação verbal do assunto devido a deficiências do layout do ambiente, como presença de pilares, espaço físico pequeno disponibilizado para as palestras, ausência de uma superfície de projeção apropriada para exibição do conteúdo.

Essa limitação estrutural não comprometeu a qualidade da ação realizada, sendo que, inclusive, colaborou para que a atividade de educação em saúde perdesse um pouco do caráter formal de palestra e adquirisse aspectos de conversação, com a atuação dos participantes do evento, o que apresenta vantagens para a integração do grupo com o tema. Nessa etapa de conversação houve discussão de alguns temas com os participantes, assim como foram sanadas algumas dúvidas, ocorrendo, dessa forma, uma retroalimentação da plateia.

Em algumas palestras houve a participação efetiva de outros profissionais da área da saúde que já atuavam com o grupo, como médicos, enfermeiros e fisioterapeutas, enfatizando o que tinha sido abordado pelo palestrante, além de complementarem com outras informações necessárias ao grupo.

No encerramento das palestras, os participantes eram informados de que, se houvesse um maior interesse em obter mais informações sobre o uso correto de medicamentos ou receber orientações específicas conforme suas situações particulares, como tratamento com medicamentos de uso contínuo, havia na universidade o Serviço de Atenção Farmacêutica (SAF) disponível para satisfazer essas e outras dúvidas relacionadas à utilização de medicamentos. Nesse momento, foram demonstradas as formas de agendamento de atendimentos no SAF e, ao mesmo tempo, os participantes que se interessaram pelo Serviço puderam repassar seu telefone para posterior contato pelos profissionais que prestavam o atendimento.

\section{Percepções}

As percepções apresentadas são frutos de oito entrevistas realizadas com os responsáveis pelos grupos nos quais as palestras foram realizadas, sendo dois profissionais da saúde e seis leigos. É importante salientar que esses últimos, além de líderes, caracterizavam-se como participantes das palestras. As entrevistas para avaliação da percepção foram realizadas no período de dois meses após a última palestra.

A entrevista iniciou-se com a apresentação dos objetivos da pesquisa e com a assinatura do termo de consentimento livre e esclarecido. Após essa etapa, foram realizados alguns questionamentos com o propósito de conhecer a percepção dos líderes sobre as palestras e, mesmo que de forma subjetiva, medir o impacto dos assuntos trabalhados no cotidiano dos indivíduos.

As entrevistas foram gravadas em áudio e, posteriormente, efetuou-se a transcrição das entrevistas e a análise dos discursos (Minayo, 2004).

Este trabalho possui aprovação no Comitê de Ética em pesquisa da Universidade do Sul de Santa Catarina.

\section{Resultados}

Foram realizadas 22 palestras no período de junho de 2006 a setembro de 2007 , com a participação de 565 indivíduos, residentes principalmente nos municípios de Tubarão e Capivari de Baixo. 0 público dessas palestras constituiu-se principalmente de mulheres com idade superior a 40 anos. As palestras tiveram duração aproximada de 90 minutos, incluindo a explanação do tema e a participação dos sujeitos da comunidade.

No transcorrer das palestras, despontaram dúvidas, comentários e depoimentos dos participantes dirigidos a assuntos não abrangidos na pré-seleção. Estes foram definidos como temas emergentes e receberam a atenção dos palestrantes que procuravam esclarecê-los aos participantes no mesmo momento. Esses temas que emergiam geralmente eram relacionados às experiências dos participantes quanto às doenças e suas formas de tratamento e controle; aos efeitos colaterais de medicamentos; à fisiopatologia de doenças diversas; aos valores de referência para exames laboratoriais; ao manejo não farmacológico de problemas de saúde; aos medicamentos manipulados; à qualidade, segurança e eficácia de medicamentos genéricos; ao acesso de medicamentos e equidade do Sistema Único de Saúde e ao uso de plantas medicinais.

Alguns depoimentos dos pacientes durante as palestras estavam relacionados com a interpretação equivocada de posologias prescritas com o número de "tomadas por dia"; a falta do entendimento de adesão terapêutica com as experiências de "parar de tomar", 
“tomar quando necessário", "alterar a posologia prescrita"; o reconhecimento das reações adversas como motivo de abandono do tratamento; o conceito de remédio e medicamento e a falta de orientação quanto aos cuidados de interações de medicamentos com alimentos, em especial com o captopril.

Além da limitação estrutural imposta pelos locais das palestras, os proponentes identificaram que os diapositivos utilizados apresentam limitação didática ao perfil dos participantes dos grupos.

\section{Percepção sobre ações de educação em saúde}

Quanto à atividade de educação em saúde, os entrevistados relataram ter sido bem desenvolvida e serviu para esclarecer muitas dúvidas sobre a utilização dos medicamentos. Muitos sujeitos que faziam tratamento com medicamentos de uso contínuo para diabetes, hipertensão e cardiopatias relataram que ainda não haviam percebido a importância de aderir completamente ao tratamento: "Antes eu achava que podia tomar o medicamento a qualquer hora, agora sei que tem hora certa [...] Melhorou porque aprendi como tomar os remédios para pressão e colesterol" (Líder de grupo participante da palestra).

A mudança de postura após a participação nas palestras ficou evidente nas opiniões apresentadas pelos participantes. Eles comentaram que antes tomavam medicamentos de forma inadequada: “Aprendi a tomar alendronato de sódio, achei que podia tomar depois do cafê" (Líder de grupo participante da palestra).

Percebeu-se que os temas abordados foram pertinentes à realidade dos participantes da atividade, pois eram facilmente contextualizados pelos ouvintes devido a sua vivência no uso de medicamentos.

Entre os temas emergentes, a automedicação foi bastante questionada, visto que vários participantes comentavam que tinham dúvidas sobre quais eram as melhores opções de medicamentos para tratar transtornos menores, como gripes, resfriados, dores musculares e constipação. No entanto, ao invés de indicar medicamentos, foi necessário orientar quanto aos riscos da automedicação, visto que os participantes eram, na sua maioria, idosos, polimedicados e com inúmeros problemas de saúde. Isso gerou uma reflexão por parte dos participantes, como observado a seguir: "Muitas pessoas, às vezes, deixam-se levar e tomam remédios sem indicação médica, assim como eu".
Dessa forma, alguns entrevistados colocaram que começaram a empregar maiores cuidados na prática de automedicação, pois muitas vezes usavam medicamentos para diminuir o sintoma apresentado, sem necessariamente observar se o mesmo era característico de transtornos menores: “Algumas faziam automedicação e após a palestra diminuíram. Tomavam remédio por indicação de outras pessoas, não tava fazendo bem... Houve uma mudança... Ficou bem esclarecido sobre automedicação [...] Eu tomava vários comprimidos para dores de cabeça, agora não tomo mais" (Líder de grupo participante da palestra).

Foi possível observar que a população atendida nesta ação possuía dúvidas a respeito de medicamentos genéricos, desde sua identificação através da embalagem até questões que envolvem a qualidade do produto e seu custo: "Importante saber que genéricos podem ser mais econômicos... Algumas (pessoas) achavam que não era igual ao original (medicamento de referência)" (Líder de grupo participante da palestra).

Outros entrevistados deixaram evidente que, a partir de sua participação na atividade de educação em saúde, foi despertada a possibilidade de buscar mais informações sobre o uso de medicamentos com o médico do serviço privado ou do serviço de saúde público e aumentou a preocupação com cuidados de rotina para manutenção da saúde: "Houve aumento na prevenção, (as pessoas do grupo) vão ao posto medir pressão, marcam exame de diabetes todo mês, estão indo no dia certo, antes eram mais descansadas" (Líder de grupo participante da palestra); "Procurei mais informações com dois médicos” (Líder de grupo participante da palestra).

Os profissionais de saúde entrevistados relataram que a atividade desenvolvida pelos farmacêuticos colaborou significativamente para que os sujeitos compreendessem, de forma clara, a necessidade de adesão aos tratamentos propostos, respeitassem os horários de tomada dos medicamentos e procurassem o médico regularmente para fazer reavaliações do estado de saúde e da evolução da doença: "As palestras foram fundamentais para que os pacientes tivessem consciência da necessidade de fazer uso dos medicamentos nas doses e horários corretos [...] compreenderam a importância e a necessidade de ter seu quadro de saúde reavaliado periodicamente por um médico" (Líder de grupo profissional da saúde). 
Além disso, um dos profissionais de saúde relatou perceber uma valorização do seu serviço pelos pacientes, pois os mesmos conseguiram compreender que esse profissional não se preocupa apenas com o acesso aos medicamentos, mas também com o uso racional daqueles dispensados: "Os pacientes se sentem mais seguros, mais valorizados. Identificam que há uma preocupação da equipe de saúde com o uso correto dos medicamentos" (Líder de grupo profissional da saúde). Ou seja, as palestras dão maior sustentação ao trabalho de orientação quanto ao uso correto de medicamentos.

Segundo outro profissional da saúde, as palestras "vieram ao encontro das necessidades do grupo, abordando conhecimentos sobre medicamentos, como horário de tomada, importância da dosagem e da automedicação... Verificou-se o aumento do interesse dos participantes na busca de informações sobre medicamentos" (Líder de grupo profissional da saúde). Esse líder concluiu a sua exposição comentando sobre a importância de trabalhar junto a outros profissionais: "Quanto mais se fala mais se conscientiza".

Quando investigado sobre o formato das palestras, os entrevistados relataram ser adequada ao tema apresentado, tanto no conteúdo quanto na forma de abordagem e tempo despendido pelas palestras.

\section{Discussão}

Um dos alicerces da Atenção Farmacêutica é a promoção do uso racional de medicamentos. Segundo a percepção dos integrantes do SAF, essa prática pode ser realizada pelo farmacêutico ao paciente durante o atendimento individual ou em grupo. De acordo com Maffacciolli e Lopes (2005), o formato de grupo possibilita o senso de inclusão, valorização e identificação entre os participantes, sendo que muitos buscam amparo diante de seus problemas de saúde. Desenvolve-se uma relação onde cada indivíduo vê-se acolhido, pois a situação alheia reflete a sua situação pessoal. 0 trabalho em grupo permite, também, o aprofundamento de discussões pelas quais se consegue ampliar conhecimentos e melhor conduzir o processo de educação em saúde, de modo que as pessoas possam superar dificuldades, obtendo maior autonomia e podendo viver mais harmonicamente com sua condição de saúde. Para Garcia e colaboradores (2006), particularmente, as atividades de grupo envolvendo idosos são bastante vantajosas por agregarem pessoas com dificuldades semelhantes, fato importante, pois a solidão é algo frequente entre os idosos.

Na visão de Alvim e Ferreira (2007), que fizeram uma análise sobre a educação popular em saúde realizada por enfermeiros, a ação do profissional de saúde na comunidade, atuando em ações educativas, pode seguir a filosofia freiriana. Segundo esses autores, “as ideias freirianas possibilitam [...], pela crítica e reflexão, a transformação de saberes dentro de um grupo que não tem o conhecimento advindo da ciência, ao mesmo tempo, também nos apropriamos do conhecimento que vem do universo do senso comum" (Alvim e Ferreira, 2007, p. 315).

Segundo Duro Martínez (2003), existem requisitos técnicos fundamentais para o desenvolvimento das ações em grupo, entre elas, a adaptação da linguagem técnica científica ao nível cultural dos integrantes dos grupos e o planejamento de um tempo de discussão após a exposição de conteúdos, para que surjam as possíveis dúvidas e o profissional de saúde possa esclarecê-las, além de permitir a troca de experiências no grupo. Esse tempo de discussão é o que enriquece o trabalho em grupo em relação a um trabalho de orientação individual.

Nesse contexto, a experiência vivenciada no SAF pode ser compreendida da mesma forma, pois foi possível passar conhecimentos à população a respeito, por exemplo, de orientação posológica, como tomar um antibiótico duas vezes ao "dia”, e, ao mesmo tempo, compreender que, para a população atendida, “dia” refere-se apenas ao período em que há claridade. Apropriando-se de conhecimentos como esse, é possível trabalhar de forma mais adequada as informações pretendidas. É importante que o profissional de saúde permita esse compartilhamento de significados, a fim de evitar desencontros entre os discursos dos pacientes e os seus próprios. Dessa forma, existe a necessidade de o profissional suplantar o discurso técnico e considerar os aspectos situacionais, emotivos e subjetivos que constituem uma realidade sui generis do grupo (Garcia e col., 2006).

Por outro lado, a discussão ocorrida após as palestras possibilitou, além da troca de experiências, o esclarecimento dos temas emergentes. Entre esses temas, ressalta-se a dificuldade para o entendimento da dife- 
rença entre medicamento e remédio, ficando evidente que o conceito de medicamento para os participantes estava atrelado ao uso de algum recurso terapêutico de forma prolongada ou contínua, indicado e acompanhado por um profissional prescritor. Enquanto o conceito de remédio estava associado a algo que seja necessário esporadicamente, sem indicação e frequência definidas pelo profissional prescritor ou extraídas de prescrições fornecidas em circunstâncias precedentes ou, ainda, indicadas por outras pessoas.

Um fato que também chamou a atenção foi a falta de compreensão da importância da adesão terapêutica por parte dos participantes das palestras, relacionado tanto ao uso de medicamentos quanto às medidas não farmacológicas necessárias em diversas doenças. Isso pode ser fruto da dificuldade de acesso à informação, que poderia ser minimizada por ações contínuas de educação em saúde promovidas por equipes interdisciplinares.

Através dos depoimentos dos profissionais foram percebidos resultados positivos, como valorização do seu trabalho junto ao serviço de saúde. Provavelmente, esse fato é consequência das estratégias que foram aplicadas nas atividades, especialmente a preocupação em aproveitar as experiências anteriores desses profissionais e conduzir os participantes a compreender a responsabilidade de cada profissional nos cuidados de saúde da comunidade. Responsabilidade que precisa ser construída pelos profissionais diariamente, no enfrentamento dos problemas da comunidade e na busca coletiva de soluções (Duarte e col., 2007).

Ressalta-se, conforme apresentado por Frota e colaboradores (2007), que quando se trabalha de forma contínua com grupos de pacientes é possível constatar uma evolução dos participantes, que pode ser identificada através do envolvimento em relação à temática trabalhada e do amadurecimento do como trabalhar coletivamente.

É necessário criar mudanças na forma como a população percebe a sua saúde e as ações para promoção e prevenção da mesma, pois, segundo Maffacciolli e Lopes (2005), a percepção pessoal funciona como uma espécie de filtro que condiciona a mensagem segundo a própria lente. As pessoas ouvem e veem conforme as suas percepções. Esses autores (Mafacciolli e Lopes, 2005) ainda afirmam que a deficiência da adesão dos pacientes pode ser relacionada ao fato de que as interações entre usuários e prestadores de serviços em saúde se configuram, atualmente, de forma superficial, em termos de comunicação. Isso pode ser causado pelo pouco tempo dedicado aos atendimentos, pelos termos utilizados ou mesmo pela forma de abordagem. Portanto, a forma de abordagem deve ser adequada à população atendida, tanto no conteúdo a ser trabalhado quanto na forma de comunicação. Dessa forma, trabalhar com a comunidade não é uma tarefa simples e exige uma preparação prévia. Segundo Duarte e colaboradores (2007), essa dificuldade em trabalhar com a comunidade é fruto de uma educação que fala "para" as pessoas e não "com" elas.

Através da análise dos discursos foi possível identificar algumas contribuições das ações de saúde na população atendida, principalmente aquelas relacionadas à adesão farmacológica, aos cuidados com a automedicação e à necessidade de acompanhamento regular do estado de saúde. Frota e colaboradores (2007), que estudaram a percepção de pais a respeito de oficinas sobre desnutrição infantil, também identificaram que as ações de educação em grupo são relevantes no sentido de contribuir para a promoção da saúde.

Segundo Alvim e Ferreira (2007), essa mudança é possível quando o sujeito, partícipe do cuidado, reflete, questiona, recusa, aceita e critica as informações transmitidas. Dessa forma, o conhecimento científico apresentado pelos profissionais de saúde é incorporado nas ações cotidianas de cuidado à saúde.

Essa ação, levando o docente a atuar junto à comunidade com os alunos, é imprescindível para a formação de um profissional voltado a ações de prevenção e promoção da saúde. Kotecki e colaboradores (2000) destacam em seu estudo que incluir atividades de educação em saúde nos currículos das Universidades pode ser um meio para encorajar o envolvimento dos profissionais de saúde nessas atividades e sua aplicação prática. Resultados semelhantes foram encontrados na pesquisa de Ciardulli e Goode (2003) e reforçam a importância da inclusão de métodos de ensino sobre a Atenção Farmacêutica e outras atividades de educação em saúde, nas diversas reformas curriculares que ocorrem por todo o país, de modo a garantir a qualificação do profissional de saúde e sua atuação efetiva na promoção em saúde, como também a importância da ação do profissional atuante no serviço público.

De forma complementar, Schommer e Cable (1996) ressaltam que a formação de profissionais farmacêu- 
ticos para a prática da Atenção Farmacêutica pode ser realizada por dois caminhos: o primeiro seria através de treinamento, com os preceptores de estágio introduzindo novas práticas; e o segundo, com os próprios acadêmicos, através de simulações de atendimento. Nessas simulações os alunos devem ser ensinados a ter uma postura ativa no desenvolvimento da relação com os pacientes.

Os proponentes perceberam que ações de educação em saúde promovidas na Universidade podem ser utilizadas como forma de aprendizagem, por meio da qual são mobilizados os conhecimentos adquiridos pelos alunos para solucionar os problemas reais apresentados no contato com os indivíduos de uma comunidade. Dessa forma, os alunos conscientizam-se de sua função de facilitadores da divulgação do conhecimento e aplicação na vida cotidiana das pessoas em sociedade. Por outro lado, há poucos estudos que demonstrem as ações de educação em saúde, por parte dos profissionais farmacêuticos, como uma importante atividade dentro das equipes de saúde (Kotecki e col., 200o), o que reforça a importância da divulgação dessa experiência.

Mesmo que as palestras tenham sido promovidas por farmacêuticos e que o principal tema abordado fossem os medicamentos, não foi identificada na fala dos entrevistados a figura do profissional farmacêutico. Observou-se que a representação desse profissional é vista como sendo a de um agente de educação em saúde que visa ao uso racional de medicamentos. Isso pode ser fruto da percepção da farmácia como estabelecimento comercial e não de saúde, e, por consequência, do farmacêutico como comerciante.

No entanto, é necessário mudar o perfil das farmácias, caracterizando-as como locais de prestação de serviços de saúde, além da comercialização de medicamentos, como abordado anteriormente. No estudo de Kotecki e colaboradores (200o) sobre a prática da educação em saúde por farmacêuticos, foram demonstradas dificuldades na realização desse serviço nos estabelecimentos farmacêuticos, como tempo restrito, falta de reembolso pelas atividades e falta de treinamento dos profissionais. Por outro lado, outros estudos, como o de Larsson (2008), apresentam outras formas de compensação por esse serviço. Esse autor, que comenta sobre a realidade do mercado farmacêutico na Suécia, hoje de domínio governamental, mostra a informação em saúde como área de competição entre os estabelecimentos que comercializam os medicamentos, colocando esta prática como forma de garantir consumidores.

Acredita-se que a mudança da prática profissional farmacêutica, com ações voltadas aos pacientes, fundamentadas na filosofia da Atenção Farmacêutica, pode ser o reinício da construção da profissão farmacêutica vinculada à saúde e tendo como maior beneficiário o paciente.

Mesmo que possam ter ocorrido limitações relacionadas ao uso de recursos didáticos para exposição dos assuntos, foi observado, através dos discursos dos participantes e responsáveis pelos grupos, que as ações de educação em saúde promovidas pelo SAF produziram um impacto positivo na população atingida. Como perspectiva para novas ações na comunidade, fica a necessidade de elaboração de materiais didáticos apropriados ao perfil dos sujeitos participantes. Um exemplo disso é relatado na experiência da Universidade Federal de Alagoas (Trezza e col., 2007) sobre a possibilidade da criação de instrumentos para o trabalho de educação popular em saúde com arte. Esses autores descrevem a possibilidade do uso de músicas e paródias, histórias e contos, poesia, dança e teatro no processo de educação. Garcia e colaboradores (2006) também reforçam que a diversificação das atividades de educação em saúde permite a redescoberta de potencialidades e, consequentemente, o aumento da autoestima, fato importante, pois nessa faixa etária (terceira idade) a depressão apresenta-se com alta prevalência.

\section{Considerações Finais}

Este trabalho apresenta uma possibilidade de ação do farmacêutico, enquanto profissional da saúde, interagindo com a comunidade e com outros profissionais da saúde. Os resultados dessa atuação apontam para a modificação das ações dos participantes quanto ao uso correto dos medicamentos e a necessidade de acompanhamento regular do estado de saúde.

As percepções dos responsáveis pelos grupos também reforçam a proposta de que o cuidado à saúde deve ser uma ação desenvolvida por vários profissionais e, nesse contexto, despertar o interesse do participante por novas informações sobre os seus problemas de saúde e seus medicamentos. 
As ações de educação em saúde têm maior impacto sobre a saúde da população quando os educadores, além do conhecimento científico, possuem habilidades para usar as experiências da população no ato de ensinar, respeitando o indivíduo e tornando-o ativo nesse processo.

\section{Agradecimentos}

Agradecemos a todos os sujeitos desta pesquisa, em especial a Coordenação da Universidade da Experiência (UNISUL - Tubarão), a Secretaria de Saúde do município de Capivari e a Secretaria de Apoio Social do município de Tubarão.

\section{Referências}

ALVIM, N. A. T.; FERREIRA, M. A. Perspectiva problematizadora da educação popular em saúde e a enfermagem. Texto Contexto Enfermagem, Florianópolis, v. 16, n. 2, p. 315-9, 2007.

BARROS, J. A. C. Políticas farmacêuticas: a serviço dos interesses da saúde? Brasília: Unesco, 2004.

CANDEIAS, N. M. F. Conceitos de educação e de promoção em saúde: mudanças individuais e mudanças organizacionais. Revista de Saúde Pública, São Paulo, v. 31, n. 2, p. 209-13, 1997.

CIARDULLI, L. M.; GOODE, J. V. Using health observances to promote wellness in community pharmacies. Journal American Pharmaceutical Association, Washington, v. 43, n. 1, p.61-8, 2003.

DUARTE, L. R.; SILVA, D. S. J. R. ; CARDOSO, S. H. Construindo um programa de educação com agentes comunitários de saúde. Interface - Comunicação, Saúde, Educação, Botucatu, v. 11, n. 23, p. 439-47, 2007.

DURO MARTÍNEZ, J.C. El discurso de los profesionales de atención primaria de la comunidad de Madrid acerca de trabajo con grupos: sobre técnicas y técnicos. Revista Española de Salud Pública, Madrid, v. 77, n. 5, p. 615-27, 2003.

FROTA, M. A.; ALBUQUERQUE, C. M.; LINARD, A.G. Educação popular em saúde no cuidado à criança desnutrida. Texto Contexto Enfermagem, Florianópolis, v. 16, n. 2, p. 246-53, 2007.
GARCIA, M. A. A.;et al. Atenção à saúde em grupos sob a perspectiva dos idosos. Revista Latino-

Americana de Enfermagem, Ribeirão Preto, v. 14, n. 2, p. 175-182, 2006.

KOTECKI, J. E.; ELANJIAN, S. I.; TORABI, M. R. Health promotion beliefs and practices among pharmacists. Journal American Pharmaceutical Association, Washington, DC, v. 40, n. 6, p. 773-9, 2000.

LARSSON, E. C. et al. Health information, an area for competition in Swedish pharmacies. Pharmacy Practice, Redondela, v. 6, n. 2, p. 74-78, 2008.

LIMA, R. T. et al. Educação em saúde e nutrição em João Pessoa, Paraíba. Revista de Nutrição, Campinas, v. 13, n. 1, p. 29-36, 2000.

MACHADO, M. F. S. et al. Integralidade, formação de saúde, educação em saúde e as propostas do SUS: uma revisão conceitual. Ciência \& Saúde Coletiva, Rio de Janeiro, v. 12, n. 2, p. 335-42, 2007.

MAFFACCIOLLI, R.; LOPES, M. J. M. Educação em saúde: a orientação alimentar através de atividades de grupo. Acta Paulista de Enfermagem, São Paulo, v. 18, n. 4, p. 439-45, 2005.

MARIN, N. et al. (org.) Assistência farmacêutica para gerentes municipais. Rio de Janeiro: Organização Pan-Americana da Saúde/Organização Mundial de Saúde, 2003. 373 p.

MARTINS, J. J., et al. Necessidades de educação em saúde dos cuidadores de pessoas idosas no domicílio. Texto Contexto Enfermagem, Florianópolis, v. 16, n. 2, p. 254-62, 2007.

MINAYO, M. C. S. O desafio do conhecimento: pesquisa qualitativa em saúde. 8. ed. São Paulo: Hucitec, 2004.

NAVES, J. O. S.; HAMANN, E. M.; SILVER, L. D. Orientação farmacêutica para DST: uma proposta de sistematização. Ciência \& Saúde Coletiva, Rio de Janeiro, v. 10, n. 4, p. 1005-14, 2005.

Organización Mundial da la Salud. Promoción del uso racional de medicamentos: componentes centrales. Genebra: OMS, 2002. Disponível em: <http://www.who.int/medicinedocs/collect/edmweb/ pdf/s4874s/s4874s.pdf >. Acesso em: 15 nov. 2007. 
Organización Mundial da la Salud. El papel del farmacéutico en el sistema de atención a la salud. In: REUNIÓN DE LA OMS, 1993, Tokio. Informe. Genebra: OMS, 1995. Disponível em: <http://www. opas.org.br/medicamentos/site/UploadArq/ops-hsshse-95-01.pdf $>$. Acesso em: 15 jul. 2008.

Organização Pan-Americana de Saúde. Consenso Brasileiro de Atenção Farmacêutica: proposta. Brasília, DF, 2002. 21 p.

SCHOMMER, J. C; CABLE, G. L. Current status of pharmaceutical care practice: strategies for education, American Journal of Pharmaceutical Education, Alexandria, v. 6o, p. 36-42, 1996.

SILVA, T. R. et al. Controle de diabetes mellitus e hipertensão arterial com grupos de intervenção educacional e terapêutica em seguimento ambulatorial de uma Unidade Básica de Saúde. Saúde e Sociedade, São Paulo, v. 15, n. 3, p.18o-9, 2006.

TREZZA, M. C. S. F.; SANTOS, R. M.; SANTOS, J. M. Trabalhando educação popular em saúde com a arte construída no cotidiano da enfermagem: um relato de experiência. Texto Contexto Enfermagem, Florianópolis, v. 16, n. 2, p. 326-34, 2007.

WINFIELD, A. J.; RICHARDS, R. M. E. Pharmaceutical practice. 2. ed. London: Churchill Livingstone, 1998.

$523 \mathrm{p}$. 